\title{
Effet protecteur du pédoncule d'ananas au cours de l'intoxication du rat Wistar par le paracétamol
}

\author{
T. J. DOUGNON *, T. M. KPODEKON, H. AHISSOU, J. GBENOU, \\ F. LOKO et A. LALEYE
}

Laboratoire de Recherches en Biologie Appliquée (LARBA), Université Abomey-Calavi (UAC), 01 BP 2009 Cotonou, Bénin.

*Auteur correspondant, Email : dougnonj@yahoo.fr

\section{RESUME}

La présente étude se propose d'évaluer l'hépatotoxicité du paracétamol chez des rats Wistar traités à l'extrait du pédoncule d'Ananas comosus. Ils ont été répartis en : Lot 1 (30 rats): animaux témoins sans paracétamol ni extrait d'Ananas comosus; Lot 2 (30 rats): $2 \mathrm{~g}$ de paracétamol par kg de poids vif et $0,06 \mathrm{~mL}$ d'extrait d'Ananas comosus par kg par jour pendant 42 jours; Lot 3 (30 rats): $2 \mathrm{~g}$ de paracétamol par $\mathrm{kg}$ de poids vif 0,12 mL d'extrait d'Ananas comosus par kg par jour pendant 42 jours; Lot 4 (30 rats): $2 \mathrm{~g}$ de paracétamol par kg de poids vif par jour et pas d'extrait d'Ananas comosus pendant 42 jours. Les taux les plus

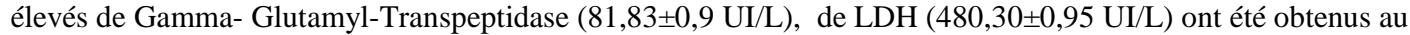
42 ème jour avec le taux le plus bas de Glutathion $(0,39 \pm 0,7 \mathrm{nM} / \mathrm{mg})$ chez les rats du lot $\mathrm{n}^{\circ} 4(\mathrm{p}<0,001)$. Les valeurs de Gamma-Glutamyl-Transpeptidase et de Lactate Deshydrogenase ont augmenté avec le paracétamol utilisé pour l'intoxication des rats alors que celle du Glutathion a sensiblement chuté. L'extrait du pédoncule d'Ananas comosus a influencé les valeurs de ces paramètres biochimiques.

(C) 2009 International Formulae Group. All rights reserved.

Mots-clés : Toxicité hépatique, paracétamol, Gamma- Glutamyl-Transpeptidase, Lactate Deshydrogenase, rat Wistar, Ananas comosus.

\section{INTRODUCTION}

Le foie joue un important rôle de métabolisation, de dégradation de la majorité des substances et ce après oxydation, réduction, conjugaison ou méthylation. Il transforme les toxiques en métabolites euxmêmes parfois très agressifs pour l'organisme. Ainsi, les pathologies hépatiques d'origine médicamenteuse sont fréquentes, polymorphes dans leur présentation et difficiles à diagnostiquer (Gruchalla, 2000). Le surdosage en Doliprane ${ }^{\circledR}$ qui contient $20 \%$ de paracétamol est fréquent car ce médicament est en vente libre et est un des produits les plus utilisés dans l'automédication. Une personne non informée sur la dose journalière prend le risque d'être confrontée dans les premières 24 heures à des cas de nausée, de vomissement, d'anorexie, de pâleur, de douleurs abdominales et de risque de cytolyse hépatique plus ou moins importante selon la quantité absorbée. Une quantité supérieure à $10 \mathrm{~g}$ par jour est extrêmement dangereuse (Lee, 2004).

Plusieurs facteurs de risque, isolés ou combinés, affectent la susceptibilité individuelle au Doliprane ${ }^{\circledR}$ et le risque d'hépatotoxicité. La présente étude se propose d'évaluer l'effet protecteur du pédoncule d'ananas au cours de l'intoxication du rat Wistar par le paracétamol.

\section{MATERIEL ET METHODES}

Matériel animal

L'étude a été réalisée sur des rats Wistar albinos mâles de $160 \mathrm{~g}$ comme poids 
moyen. Les animaux (120) ont été répartis en plusieurs groupes de 30 rats chacun. Ils avaient libre accès à l'eau et à la nourriture. La température de l'animalerie était maintenue à $22{ }^{\circ} \mathrm{C}$, une hygrométrie de $60 \%$ et une photopériode de 12 heures/24.

\section{Ananas frais}

L'Ananas comosus, variété «pain de sucre» provenait de la Ferme d'Application du Lycée Agricole Mèdji de Sékou, situé dans la Commune d'Allada, Département de l'Atlantique au Bénin.

\section{Extrait d'Ananas comosus}

L'ananas frais a été transformé pour obtenir l'extrait qui a été utilisé pour traiter les rats Wistar intoxiqués au Doliprane ${ }^{\circledR}$.

Les paramètres biochimiques, GammaGT, Glutathion et Lactate Deshydrogenase ont été dosés selon le protocole indiqué dans les Kits.

\section{Obtention de la dose toxique de Doliprane ${ }^{\circledR}$}

Les comprimés de Doliprane ${ }^{\circledR}$ à $20 \%$ du paracétamol ont été réduits en poudre. La $\mathrm{DL}_{50}$ a été recherchée pour les rats intoxiqués au Doliprane ${ }^{\circledR}$. En considérant $2500 \mathrm{mg}$ de Doliprane ${ }^{\circledR}$ pour $1 \mathrm{~kg}$ de poids vif (PV) de rat, il a été constaté la mort de 5 rats sur 10 choisis pour l'expérience. La dose de Doliprane ${ }^{\circledR}$ a été alors réduite jusqu'à $2000 \mathrm{mg}$ pour $1 \mathrm{~kg}$ de PV de rat pour l'induction de la toxicité hépatique avec le Doliprane ${ }^{\circledR}$.

Quant aux doses de l'extrait de l'ananas, les quantités de cet extrait ont été corrélées à la quantité d'ananas frais utilisé pour corriger des lésions hépatiques de rat Wistar intoxiqué avec le paracétamol au cours de précédentes études.

\section{Les groupes d'animaux}

Lot 1 (30 rats): animaux témoins qui n'ont ni du Doliprane $囚$, ni d'extrait de l'Ananas comosus.

Lot 2 (30 rats): les rats ont reçu par voie orale $2 \mathrm{~g}$ de Doliprane ${ }^{\circledR}$ par $\mathrm{kg}$ de poids vif et un traitement de $0,06 \mathrm{~mL}$ de l'extrait d'Ananas comosus par kg par jour pendant 42 jours

Lot 3 (30 rats): les rats ont reçu par voie orale $2 \mathrm{~g}$ de Doliprane ${ }^{\circledR}$ par $\mathrm{kg}$ de poids vif et un traitement de $0,12 \mathrm{~mL}$ de l'extrait d'Ananas comosus par kg par jour pendant 42 jours

Lot 4 (30 rats): les rats ont reçu par voie orale $2 \mathrm{~g}$ de Doliprane ${ }^{\circledR}$ par $\mathrm{kg}$ de poids vif par jour pendant 42 jours et n'ont pas reçu de traitement à base d'extrait d'Ananas comosus.

\section{Obtention de l'extrait d'Ananas comosus}

Le procédé d'extraction de l'extrait concentré de l'Ananas comosus comporte plusieurs étapes telles que: le broyage, l'extraction, la filtration la décantation, la centrifugation et la concentration.

\section{Screening phytochimique de l'ananas}

Les différents groupes chimiques de l'ananas ont été recherchés. Les flavonoïdes auxquels on attribue les effets biologiques de l'extrait d'Ananas comosus ont été recherchés et séparés par chromatographie sur couche mince, réalisée selon les méthodes de Park et al. (2001). Le système solvant utilisé a été l'éthanol à $95^{\circ}$ et l'eau distillée. Les bandes séparées ont été observées à l'UV à $254 \mathrm{~nm}$ et $336 \mathrm{~nm}$; la chromatographie sur papier a été réalisée sur papier Watman $\mathrm{n}^{\circ} 3$ dans l'acide acétique $15 \%$ et la délimitation des différentes bandes de produits présents dans le chromatogramme sous lumière de Wood à 360-366 nm.

Dans le méthanol neutre, les composés flavoniques absorbent dans deux régions différentes du spectre ultra-violet, entre 300 et $385 \mathrm{~nm}$ et entre 250 et $280 \mathrm{~nm}$.

\section{Les dosages biochimiques}

Les dosages biochimiques de GammaGlutamyl-Transférase et de la Lactate Déshydrogénase (LDH) ont été réalisés en utilisant des kits de commerce prêts à l'emploi (kits Biomérieux) et les lectures d'absorbance ont été réalisées avec un spectrophotomètre (Spectronic 601). Le glutathion hépatique a été dosé par spectrophotométrie selon la méthode de Park et al. (2001).

\section{Méthodes statistiques utilisées}

Après le traitement, les données ont été soumises à l'Analyse de Variance sous le Logiciel Statistica 6.0 (1998). 
RESULTATS

Concentration de Gamma-Glutamyl Transpeptidase chez les rats intoxiqués au Doliprane ${ }^{\circledR}$ et traités avec Ananas comosus : figure 1

La valeur la plus élevée $(30 \pm 0,00 \mathrm{U} / \mathrm{L})$ obtenue au J0 a été obtenue avec les animaux du lot $n^{\circ} 2$ mais sans différence significative avec le taux obtenu chez les rats des autres lots $(p>0,05)$. Toutefois, les taux de ce paramètre biochimique ont subi des variations en hausse à partir du $7^{\text {ème }}$ jour; la valeur la plus élevée $(81,83 \pm 0,9 \mathrm{U} / \mathrm{L})$ étant obtenue au $42^{\text {ème }}$ jour avec les rats du lot $\mathrm{n}^{\circ} 4(\mathrm{p}<0,001)$.

Concentration de Glutathion chez les rats intoxiqués au Doliprane ${ }^{\circledR}$ et traités avec Ananas comosus : figure 2

Les niveaux les plus élevés de Glutathion $(1,83 \pm 0,01$ et $1,83 \pm 0,3 \mathrm{nM} / \mathrm{mg})$ ont été obtenus avec les rats des lots $\mathrm{n}^{\circ} 2$ et $\mathrm{n}^{\circ} 4$ sans différence significative avec les valeurs obtenues au niveau des autres lots $(p>0,05)$. Une chute du taux de Glutathion a commencé dès le $7^{\text {ème }} \mathrm{j}$ pour atteindre le niveau le plus bas $(0,39 \pm 0,7 \mathrm{nM} / \mathrm{mg})$ chez les rats du lot $\mathrm{n}^{\circ} 4$ contrairement aux valeurs de ce paramètre biochimique chez les rats des autres lots $(\mathrm{p}<0,001)$.

Concentration de Lactate Déshydrogénase (LDH) chez les rats intoxiqués au Doliprane ${ }^{\circledR}$ et traités avec Ananas comosus : figure 3

La valeur de LDH la plus élevée $(310,73 \pm 1,31)$ a été obtenue au J0 chez les rats des lots $n^{\circ} 1$ et $n^{\circ} 3$ avec des variations très hautement significatives quand on les compare aux résultats obtenus avec les rats des autres lots $(p<0,001)$. Le taux de LDH a subi une augmentation dès le $7^{\text {ème }} \mathrm{j}$ pour atteindre contrairement aux valeurs des rats des autres lots, la valeur la plus élevée de $480,30 \pm 0,95 \mathrm{U} / \mathrm{L}$ au $42^{\text {ème }} \mathrm{j}(\mathrm{p}<0,001)$.

\section{DISCUSSION}

Taux de Gamma- Glutamy-Transpeptidase

La Gamma- Glutamy-Transpeptidase est un marqueur du fonctionnement hépatique; son taux chez les animaux qui n'ont pas été intoxiqués n'a pas varié de façon significative. $\mathrm{La}$ valeur de $29,35 \pm 0,1 \mathrm{UI} / \mathrm{L}$ trouvée au cours de la présente étude est moins élevée que celle proposée par (Kaneko,
1989). Cet auteur avait rapporté une valeur $32,35 \pm 0,4 \mathrm{UI} / \mathrm{L}$ chez des rattes. Le sexe des animaux aurait influencé ce résultat. Par ailleurs, la valeur de $81,83 \pm 0,91 \mathrm{UI} / \mathrm{L}$ obtenue au cours de notre étude est largement

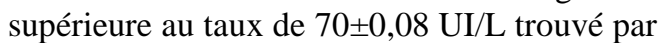
Lahouel et al. (2004) chez des rats intoxiqués avec du paracétamol à raison de $200 \mathrm{mg} / \mathrm{kg}$ de poids vif par jour. Les taux les plus élevés sont rencontrés chez les rats du lot $n^{\circ} 4$. Une augmentation de cette enzyme pourrait objectiver une cholestase mais aussi une éventuelle cytolyse en l'absence de toute cholestase (Farougou, 1992). Les altérations histologiques du foie constatées par Dougnon et al. (2006) au cours d'une étude antérieure confirment cette thèse. L'augmentation modérée de cette enzyme dont le taux n'a pas subi de grandes variations chez les rats des lots $n^{\circ} 2$ et $n^{\circ} 3$ pourrait s'expliquer par l'efficacité réparatrice de Ananas comosus dont l'extrait a été utilisé à la dose de $0,06 \mathrm{ml}$ et $0,12 \mathrm{ml}$ par $\mathrm{kg}$ de poids vif par jour pendant 42 jours.

\section{Taux de Glutathion}

Le taux de glutathion hépatique a subi une diminution très hautement significative $(0,39 \mathrm{nM} / \mathrm{mg}$ de protide) chez les animaux intoxiqués au Doliprane ${ }^{\circledR}$ et qui n'ont pas été traités par Ananas comosus au J42. Une tendance similaire de diminution du taux de Glutathion a été observée par Lahouel et al. (2004). En effet, la chute de ce paramètre biochimique constatée par ces auteurs, a été plus sévère $(0,30 \mathrm{nM} / \mathrm{mg}$ de protide) chez des rats intoxiqués avec $200 \mathrm{mg}$ de Paracétamol. Cette différence de taux de glutathion pourrait s'expliquer par le fait qu'en dehors de l'espèce, de la substance, de l'état physiologique de l'animal, de la dose administrée, l'impact nocif des xénobiotiques, varie aussi en fonction d'autres facteurs comme la durée et le mode d'exposition (Vialia, 1998).

La baisse de ce paramètre biochimique est liée à l'effet toxique du Paracétamol. En effet, c'est au cours du métabolisme de ce médicament reconnu comme xénobiotique que le système enzymatique cytochrome $\mathrm{P} 450$ intervient (Guengerich, 1986). C'est ainsi que le Paracétamol est métabolisé par le cytochrome P450 pour donner un métabolite réactif ( $\mathrm{N}$-acétyl-p-benzoquinone-imine) 


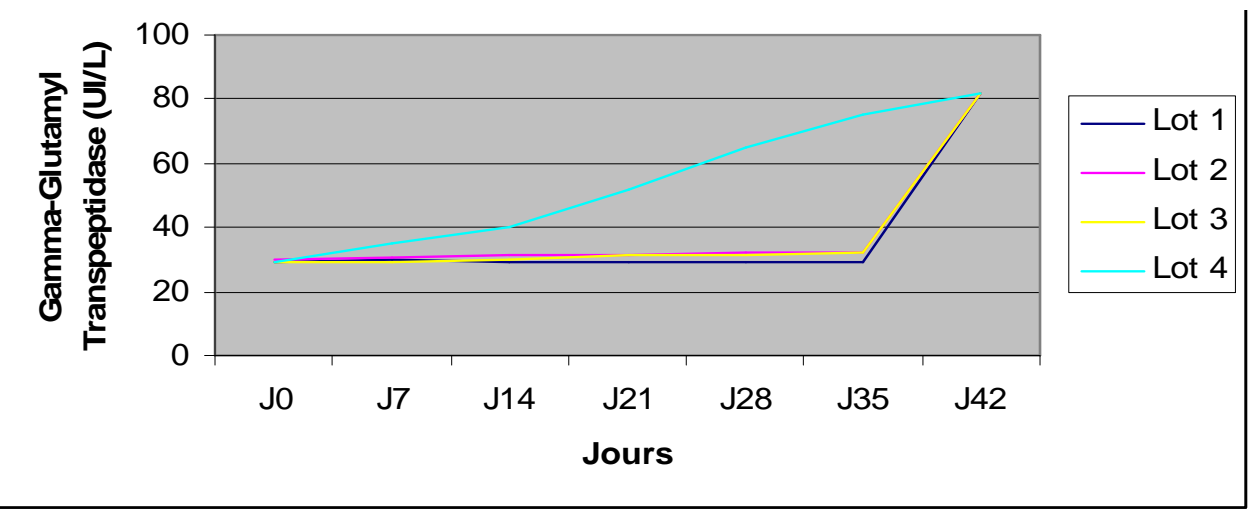

Figure 1: Evolution des niveaux de Gamma-Glutamyl Transpeptidase chez les rats Wistar. Lot $\mathbf{n}^{\circ} \mathbf{1}$ : Témoin, sans Doliprane ${ }^{\circledR}$ ni extrait d'ananas. Lot $\mathbf{n}^{\circ} \mathbf{2}$ : Doliprane ${ }^{\circledR}$ à raison de $2 \mathrm{~g} / \mathrm{kg}$ de poids vif (PV) par jour puis 0,06 $\mathrm{ml}$ par $\mathrm{kg}$ de PV pendant $42 \mathrm{j}$. Lot $\mathbf{n}^{\circ} \mathbf{3}$ : Doliprane ${ }^{\circledR}$ à raison de $2 \mathrm{~g} / \mathrm{kg}$ de $\mathrm{PV}$ par jour puis $0,12 \mathrm{ml}$ par $\mathrm{kg}$ de $\mathrm{PV}$ pendant $42 \mathrm{j}$. Lot $\mathrm{n}^{\circ} 4$ : Doliprane ${ }^{\circledR}$ à raison de $2 \mathrm{~g} / \mathrm{kg}$ de $\mathrm{PV}$ par jour pendant 42 jours sans aucun traitement avec l'extrait d'ananas.

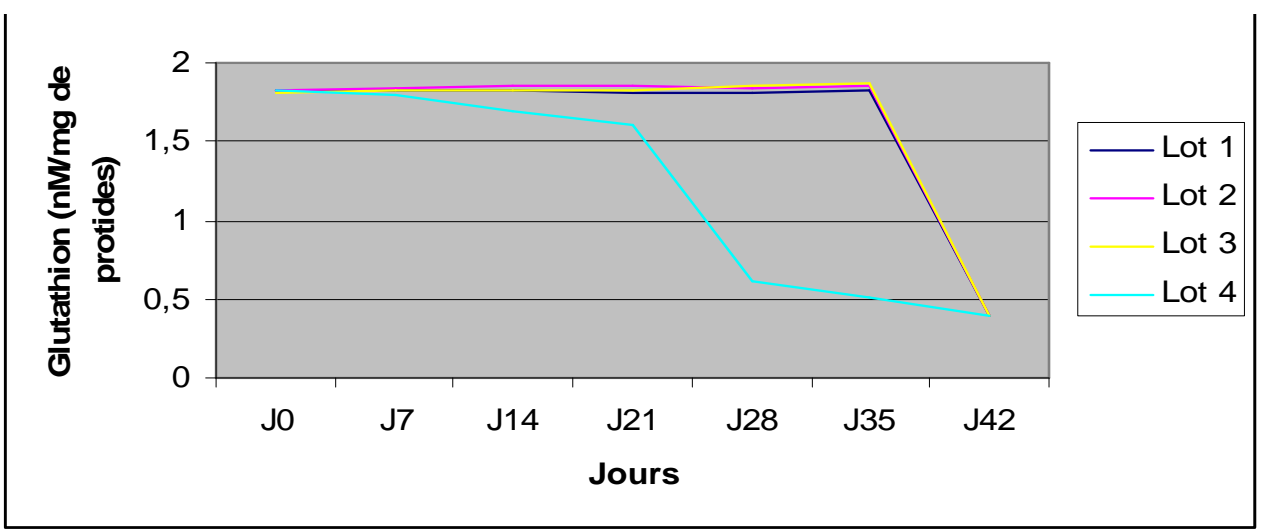

Figure 2 : Evolution des niveaux de Glutathion (nM/mg de protides) chez les rats Wistar. Lot $\mathbf{n}^{\circ} \mathbf{1}$ : Témoin, sans Doliprane® ni extrait d'ananas. Lot ${ }^{\circ} 2$ : Doliprane ${ }^{\circledR}$ à raison de $2 \mathrm{~g} / \mathrm{kg}$ de poids vif (PV) par jour puis 0,06 $\mathrm{ml}$ par $\mathrm{kg}$ de PV pendant $42 \mathrm{j}$. Lot $\mathbf{n}^{\circ} \mathbf{3}$ : Doliprane ${ }^{\circledR}$ à raison de $2 \mathrm{~g} / \mathrm{kg}$ de $\mathrm{PV}$ par jour puis $0,12 \mathrm{ml}$ par $\mathrm{kg}$ de $\mathrm{PV}$ pendant $42 \mathrm{j}$. Lot $\mathbf{n}^{\circ} 4$ : Doliprane ${ }^{\circledR}$ à raison de $2 \mathrm{~g} / \mathrm{kg}$ de $\mathrm{PV}$ par jour pendant 42 jours sans aucun traitement avec l'extrait d'ananas.

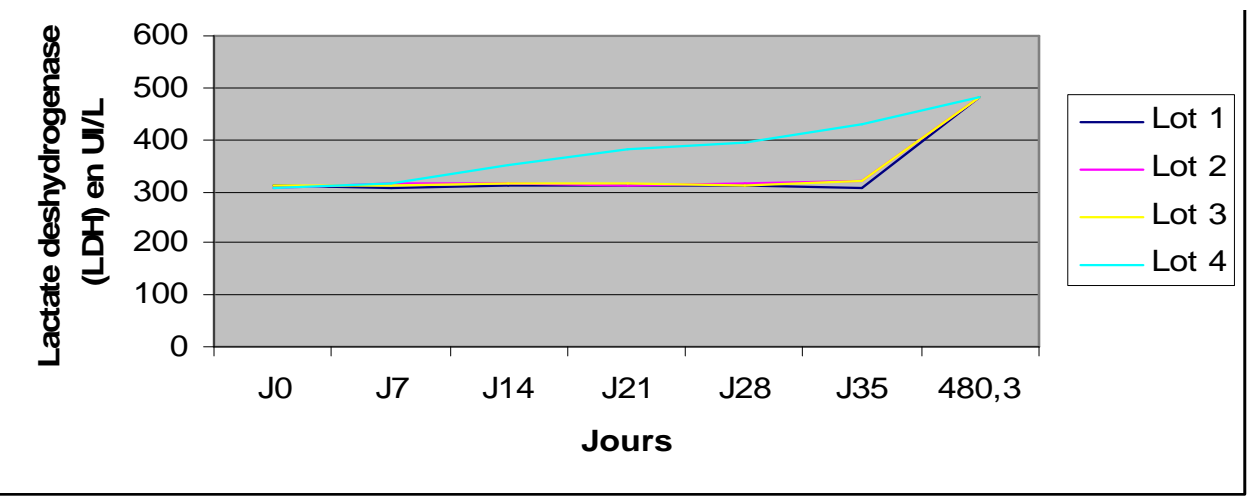

Figure 3: Evolution des niveaux de Lactate Deshydrogenase chez les rats Wistar. Lot no 1 : Témoin, sans Doliprane ${ }^{\circledR}$ ni extrait d'ananas. Lot $\mathbf{n}^{\circ} \mathbf{2}$ : Doliprane ${ }^{\circledR}$ à raison de $2 \mathrm{~g} / \mathrm{kg}$ de poids vif (PV) par jour puis $0,06 \mathrm{ml}$ par $\mathrm{kg}$ de $\mathrm{PV}$ pendant $42 \mathrm{j}$. Lot $\mathbf{n}^{\circ} \mathbf{3}$ : Doliprane ${ }^{\circledR}$ à raison de $2 \mathrm{~g} / \mathrm{kg}$ de $\mathrm{PV}$ par jour puis $0,12 \mathrm{ml}$ par $\mathrm{kg}$ de $\mathrm{PV}$ pendant $42 \mathrm{j}$. Lot $\mathbf{n}^{\circ} 4$ : Doliprane $®$ à raison de $2 \mathrm{~g} / \mathrm{kg}$ de $\mathrm{PV}$ par jour pendant 42 jours sans aucun traitement à l'extrait d'ananas. 
conduisant à la nécrose hépatique par péroxydation des lipides membranaires. Selon Lahouel et al. (2004), cette toxicité est observée au-delà des doses supérieures à 200 $\mathrm{mg} / \mathrm{kg}$ par jour. Par ailleurs, la cellule dispose pour sa protection, du glutathion. C'est le principal anti-oxydant propre de la cellule en se liant par son pôle SH aux métabolites toxiques. L'exemple le plus démonstratif étant l'intoxication au Paracétamol dont la toxicité hépatique n'apparaît qu'avec la déplétion du foie en glutathion (Lahouel et al., 2004). On admet aussi aujourd'hui que la formation de métabolites réactifs consomme du glutathion quand elle est importante et que cela aboutit à une déplétion du glutathion, ce qui a pour principale conséquence la péroxydation lipidique (Settaf et al., 2000) et une oxydation des groupes thiols des protéines (Bridger et al., 2000 ; Mofredj et al., 1999).

Il a été constaté par ailleurs que les taux de glutathion ne sont pas statistiquement différents chez les rats des lots $n^{\circ} 2, n^{\circ} 3$ et $n^{\circ} 1$. Ceci pourrait s'expliquer par le rôle antioxydant d'Ananas comosus. En effet, ce fruit contient, grâce aux résultats du screening pharmacologique réalisé au cours de cette étude, des flavonoïdes et de la vitamine C. Ils ont la capacité de capturer et de désactiver les radicaux libres. Selon Siess et al. (2000), ils agissent par empêchement de la fixation des radicaux libres sur l'ADN, par l'activation du système de détoxication et par protection des parois capillaires (Kawabata et al., 1990).

\section{Taux de Lactate-Déshydrogénase (LDH)}

Le taux de la LDH a subi de grandes variations dès le $\mathrm{J} 0$. La concentration la plus élevée $(480,30 \pm 0,95)$, a été obtenue avec les animaux du lot $\mathrm{n}^{\circ} 4$ avec une différence très hautement significative par rapport au taux de LDH des rats des lots $n^{\circ} 1, n^{\circ} 2$ et $n^{\circ} 3$ au J42. La valeur de $310,73 \pm 1,31$ de $\mathrm{LDH}$ chez les rats non intoxiqués au cours de la présente étude diffère de la valeur de la LDH dosée par Boukerche et al. (2007). Le taux le plus élevé de la LDH chez les rats intoxiqués et qui n'ont reçu aucun traitement à base d'Ananas comosus serait lié à la modification des protéines dans le foie des rats intoxiqués par le paracétamol (Bermeyer, 1980). L'accumulation de pyruvate au niveau du foie a été proposée pour expliquer l'augmentation de la LDH qui catalyse la réduction de ce dernier en Lactate (Boukerche et al., 2007). Dans ce cas, on pourrait suggérer que la forte activité enzymatique de la GlutamateOxaloacétique transaminase (GOT), de la Glutamate Pyruvate transaminase (GPT) et de la phosphatase alcaline et publiée par Dougnon et al. (2008), est liée à l'effet hépatotoxique du paracétamol chez les rats du lot $\mathrm{n}^{\circ} 4$; le foie étant le principal organe de détoxication des xénobiotiques. Il contient la plupart des enzymes pour réussir cette mission. L'élévation du taux de LDH au niveau des rats du lot $\mathrm{n}^{\circ} 4$ suppose la forte hémolyse au niveau du foie de ces rats. Même si les taux de LDH obtenus avec les rats des lots $n^{\circ} 2$ et $n^{\circ} 3$ n'ont pas dépassé celui obtenu chez les animaux du lot $\mathrm{n}^{\circ} 1$, on observe une baisse du taux de ce paramètre biochimique au niveau de ces rats Wistar.

\section{Conclusion}

De l'analyse des résultats de la présente étude, il ressort que:

Le Doliprane $\AA$ utilisé à raison de $2 \mathrm{~g} / \mathrm{kg}$ de poids vif par jour pendant 42 jours a induit une toxicité hépatique chez les rats albinos Wistar par une variation des taux de GammaGlutamyl Transpeptidase, de la Lactate Deshydrogenase et du Glutathion.

Les taux des paramètres biochimiques (Gamma-Glutamyl Transpeptidase, Lactate Deshydrogenase et Glutathion ont été corrigés par l'extrait du pédoncule d'ananas utilisé à raison de $0,06 \mathrm{ml} / \mathrm{kg}$ ou de $0,12 \mathrm{ml} / \mathrm{kg}$ de poids vif $(\mathrm{pV})$ par jour pendant 42 jours.

Les résultats de notre étude révèlent une fois encore le rôle hépato-protecteur des flavonoïdes et autres substances antioxydantes que renferme l'Ananas comosus ce qui appelle à une valorisation de ce produit alimentaire.

\section{REFERENCES BIBLIOGRAPHIQUES}

Bergmeyer H. 1980. Dietary nitrate in man. Clin. Chem. Acta., 105: 147-52.

Boukerche S, Aouacheri W, Saka S. 2007. Les effets toxiques des nitrates: étude biologique chez l'homme et chez l'animal. Ann. Biol. Clin., 65(4): 38.

Bridger S, Henderson K, Glucksman E, Ellis A, Henry J. 2000. Death from low dose Paracetamol poisoning. Eur. J. Pharmacol., 316: 1724-5.

Farougou S. 1992. Contribution à 
l'établissement des valeurs usuelles sériques chez l'aulacode mâle adulte thryonomys swinderianus. Thèse de doctorat de médecine vétérinaire. EISMV. Dakar, 102p.

Dougnon TJ, Kpodékon TM, Lalèyè A, Adjagba M, Darboux BR. 2006. Effet de Ananas comosus, (variété "Pain de sucre") sur les lésions hépatiques dues à l'intoxication au paracétamol chez le rat Wistar. Journal de la Société de Biologie Clinique, 10: 47-50.

Dougnon TJ, Kpodékon TM, Adjagba M. 2008. Biochemical effectiveness in liver detoxication of fresh pineapple (Ananas comosus) with the Wistar rats, previously intoxicated by Doliprane ${ }^{\circledR}$. Journal of Cell and Animal Biology, 2(2): 31-35.

Gruchalla RS. 2000. Clinical assessment of drug-induced disease. Lancet, 356: 150511.

Guengerich P. 1986. Mammalian Cytochromes P450. CRC press: Florida.

Kaneko JJ. 1989. Chemical Biochemistry of Domestic Animals (4 ${ }^{\mathrm{eme}}$ édn). Academic press Inc.: California; 932.

Kawabata TT, Chapman MY, Kim DH, Stevens WD, Holsapple MP. 1990. Mechanisms of in vitro immunosuppression by hepatocyte-generated cyclophosphamide metabolites and 4hydroxycyclophosphamide. Biochem.
Pharmacol., 40(5): 9-27.

Lahouel M, Boulkour S, Segueni N, Fillastre JP. 2004. Effet protecteur des flavonoïdes contre la toxicité de la vinblastine, cyclophosphamide et du Paracétamol par inhibition de la peroxydation lipidique et augmentation du glutathion hépatique. $J$. Pat. Bio., 10: 314-32.

Lee WM. 2004. Acetaminophen and the US acute liver failure study group: Lowering the risks of hepatic failure. Hepatology, 40: 6-9.

Mofredj A, Cadranel J, Darchy B, Barbare J, Cazier A. 1999. Toxicité hépatique du Paracétamol à dose thérapeutique chez le sujet éthylique chronique. Ann. Med. Int., 150: $507-511$.

Parks JH, Lee HH, Ju SK, Lee MK, Kim KL. 2001. Protein Exp. Purif., 22(1): 60-69.

Settaf M, Zahidy A, Elimadi R, Sapena I, Abd A. 2000. S-15176 reduces the hepatic injury in rats subjected to experimental ischemia and reperfusion. Eur. J. Pharmacol., 406: 281-292.

Siess M. 2000. Le Bon A, Canivenc-lavier M, Susch M. Mechanisms involved in the chemoprevention of flavonoids. Biofactors, 12(4): 193-199.

Vialia A. 1998. Eléments de Toxicologie. Editions Médicales Internationales: Paris; 24. 